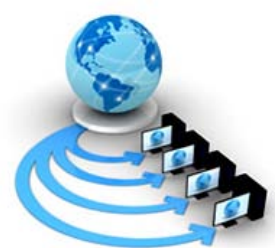

Volume 8, No. 7, July - August 2017

International Journal of Advanced Research in Computer Science

RESEARCH PAPER

\author{
Available Online at www.ijarcs.info
}

\title{
TRADITIONAL FEATURE DETECTION METHOD USING SPECTRUM SENSING WITH CHANNEL STATE DIVERSITY (SSCD) ALGORITHM
}

\author{
Karamjot Kaur \\ Department of ECE \\ Baba Banda Singh Bahadur Engineering College \\ Fatehgarh sahib, Punjab, India
}

\author{
Raminder Singh Uppal \\ Department of ECE \\ Baba Banda Singh Bahadur Engineering College \\ Fatehgarh Sahib, Punjab, India
}

\begin{abstract}
The rapidly growing number of wireless communication system devices had led to massive increase in radio traffic density, resulting in the shortage of available spectrum. There are various spectrum sensing techniques. Energy Detection (ED) and Feature Detection (FD) methods exploit the statistical periodicity of transmission signals and perform better under low Signal to Noise (SNR) ratio. The objective of this paper is to analyze the negative impact of fast time- varying channels on the traditional FD sensing methods. In result of which an algorithm is proposed named, "Spectrum Sensing with Channel State Diversity (SSCD)". SSCD algorithm identifies the features of primary users under fast time varying channels.
\end{abstract}

Keywords: Spectrum Sensing, Channel-State Diversity, Feature Detection, Cognitive Radio.

\section{INTRODUCTION}

Wireless technology is the backbone of future computing world and wireless spectrum resources had faced more and more user demand from the last decade. The shortage of spectrum resource is a challenging issue. Cognitive Radio (CR) offers promising solution that aim to improve the spectrum utilization. Earlier to satisfy the user need Federal communication commission (FCC) had adopted Fixed Spectrum Allocation (FSA) policy. But later it was observed that some of the frequency bands remain unoccupied for long time, some are partially occupied and remaining bands are heavily used. So there is a scope to improve the spectrum efficiency [1]. To solve this dynamic spectrum allocation strategy was adopted in which the part of spectrum which is not used by primary users is used by the secondary users and referred as Cognitive Radio (CR) technology. The concept of CR was introduced in 1999 by Joseph Mitola. A CR network is composed of primary users (PU) and cognitive/secondary users (SU) [2].

Spectrum sensing techniques means sensing the spectrum and finds the unused band and shares it with SU while avoiding the interference with the spectrum occupied by the PU. Common spectrum sensing techniques can be categorized into different types such as Energy Detection (ED), Feature Detection (FD), Matched Filtering Detection, Waveform based sensing, Radioidentification based sensing etc. ED and FD are mostly used. $\mathrm{ED}$ is the most common spectrum sensing technique because of its low complexity and computational cost [3]. It is performed by comparing the received energy of a signal against predefined threshold in order to determine the presence and absence of the user in the frequency band of interest. But it has poor performance under low SNR conditions. FD relies on the fact that most communication signals have periodicity in their statistics. FD differentiated the modulated signal from the additive noise. In order to eliminate the noise interference FD need adequate size of observation window in sensing process [4]-[6]. FD detects the presence of user even under low SNR conditions. When the channel coherence time is shorter than the observation window, the identification of statistical features will be distorted and hinders the accuracy of FD techniques [7] [8].

In this paper, we will try to find out the solution for effectively identifying the statistical features under fast-time varying channels. The negative effect of fast time-varying channel on traditional FD sensing techniques will be examined and find the effect of channel-state diversity. Based on this diversity effect an algorithm is proposed name, "Spectrum Sensing with Channel State Diversity (SSCD)”. This algorithm captures the feature branches with different phases and accumulates them to form the targeted feature. It will be demonstrated that SSCD algorithm offers better detection accuracy than traditional FD with fixed window (FDFW) under fast time-varying channels [9].

\section{BACKGROUND}

Firstly we analyzed the performance of traditional FD techniques under Fast-time varying channels. The method for FD sensing are Spectral Correlation Function (SCF) and cyclic autocorrelation function (CAF), both are based on signal correlation function [10]. Signal correlation Function is used as an indicator to reveal the influence of fast time varying channels.

Sensing judgment can be executed as:

$$
\begin{aligned}
& \mathrm{D}_{0}:|\mathrm{F}|<\Gamma \\
& \mathrm{D}_{1}:|\mathrm{F}| \geq \Gamma
\end{aligned}
$$

Where $D_{0}$ and $D_{1}$ represents the presence and absence of primary user transmission, respectively. $\Gamma$ represents the threshold coefficient [1].

Lets consider an illustration to show the negative effect of the fast time varying channels. In the illustration, we have taken 512 sub-carriers, under $\mathrm{H}_{1}$ with $\mathrm{L}=10, \mathrm{SNR}=-5 \mathrm{db}$ and $\Delta=160$. Fig. 1 and fig. 2 represents the accumulation of signal correlation components for coverages of two sub-pieces with different static channel states. 


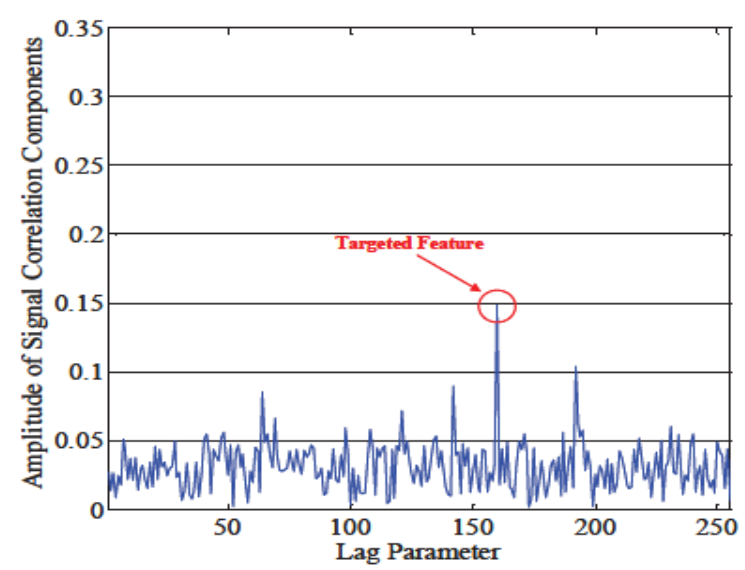

Fig. 1 Sub Piece 1

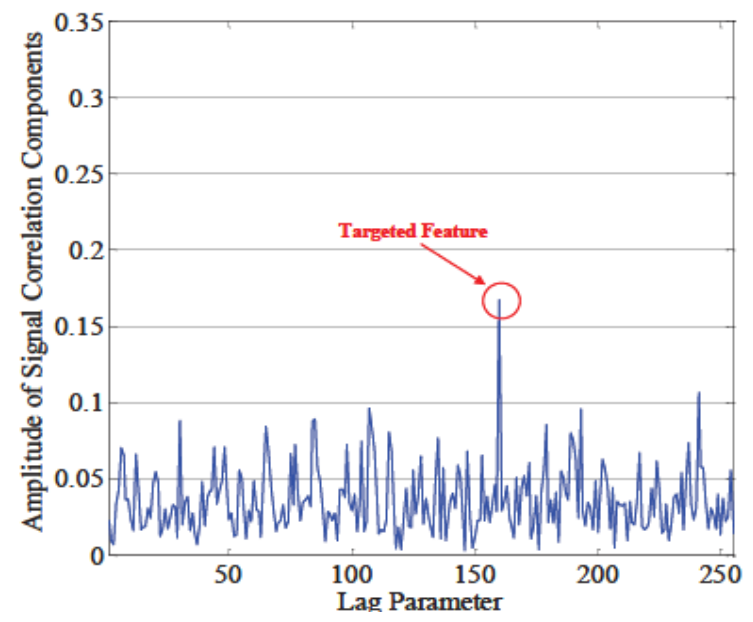

Fig. 2 Sub Piece 2

Fig 3 shows the result when two sub-pieces are combined under traditional FD sensing methods. It shows that the targeted peak loses it preponderence.

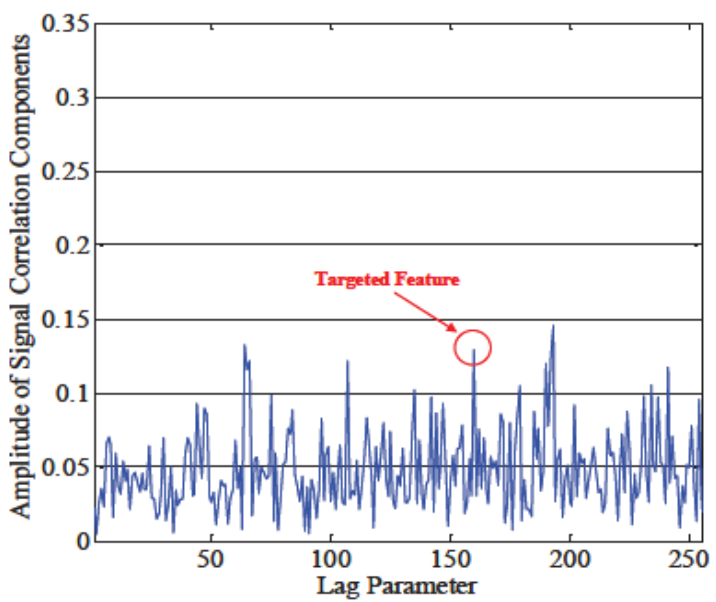

Fig. 3 Combination two sub-pieces using tarditional FD sensin

\section{SPECTRUM SENSING WITH CHANNEL STATE DIVERSITY}

According to the previous discussion, Fast-time varying channels cannot extract feature branches well, so that the sensing accuracy is not satisfactory [11]. We proposed SSCD algorithm in which we infer that if all the feature-branches are effectively captured and accumulated from different phases, not only the sensing accuracy is protected, but also channelstate diversity can be achieved. Such diversity effect can be theoretically embodied as follows: when part of featurebranches within an observation window is distorted by deep fading channel states, the negative effect will be contained by other positive Feature-branches [12] [13]. Therefore, channelstate diversity reverses fast time-varying channels into a benefit for sensing issues. Assuming that channel coherence time is known so that the observation window with the dynamic channel can be separated into sub-pieces with several static channel states.

In case of SSCD, peak of targeted feature exploits the amplitude of feature branches from two sub-pieces as shown .

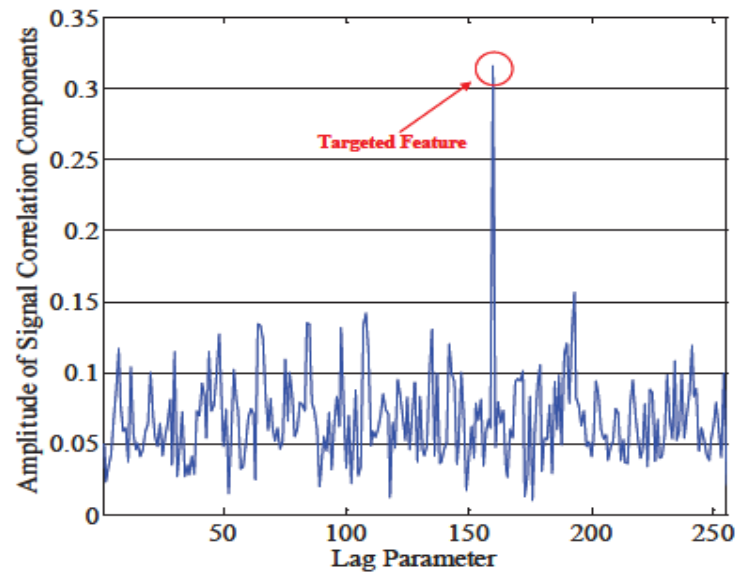

Fig. 4 Combination two sub-pieces using channel state diversity

\section{RESUlts AND Discussions}

SSCD algorithm is testified through numerical results.

Simulation parameters are listes as:

Carrier frequency $=2.5 \mathrm{GHz}$, FFT size $=512$, CP Length $=\mathrm{N} / 4$, coding rate $=1 / 2$ and the observation window size $=10$. The modulation used is QPSK and rayleigh fading is used [1].

Before sensing the threshold should be adjusted to the required probability of false-alarm $\left(\mathrm{P}_{\mathrm{f}}\right)$. Detection threshold can be expressed as:

$$
\gamma=\Gamma / \mathrm{N}_{0}^{2}
$$

$\mathrm{N}_{0}{ }^{2}$ is the noise energy base. $\mathrm{P}_{\mathrm{f}}$ is only decided by $\gamma$, which provides practical designing and regulations. Under SSCD algorithm, $\mathrm{P}_{\mathrm{f}}$ show variations, which is related to window separation strategy during the sub-piece capturing.

Fig. 5 shows the probability of false-alarm under different channel varying rates. FDFW always keep the same profile and the curves overlap with each other. FDFW always maintain a same probability of false alarm as:

$$
\begin{aligned}
P_{f} & =\operatorname{Pr}\left(D_{1} \mid H_{0}\right) \\
& =\operatorname{Pr}\left(\left|\frac{1}{L M} \sum_{n=0}^{L M-1} v(n) v^{*}(n+\Delta)\right| \geq \Gamma\right)
\end{aligned}
$$

SSCD algorithm has various profiles under different scenarios. In this there will be a need of higher $\gamma$ with the increase of 
channel varying rate. Probablity of false alarm under this condition is given as:

$$
\begin{aligned}
& \operatorname{Pf}=\operatorname{Pr}\left(\frac { 1 } { L M } \left\{\sum_{\psi=0}^{\Psi-2}\left|\sum_{n=\psi \hat{L} M}^{(\psi+1) \hat{L} M-1} v(n) v^{*}(n+\Delta)\right|\right.\right. \\
& \left.\left.+\left|\sum_{n=(L-\dddot{L}) M}^{L M-1} v(n) v^{*}(n+\Delta)\right|\right\} \geq \Gamma\right) .
\end{aligned}
$$

(4)

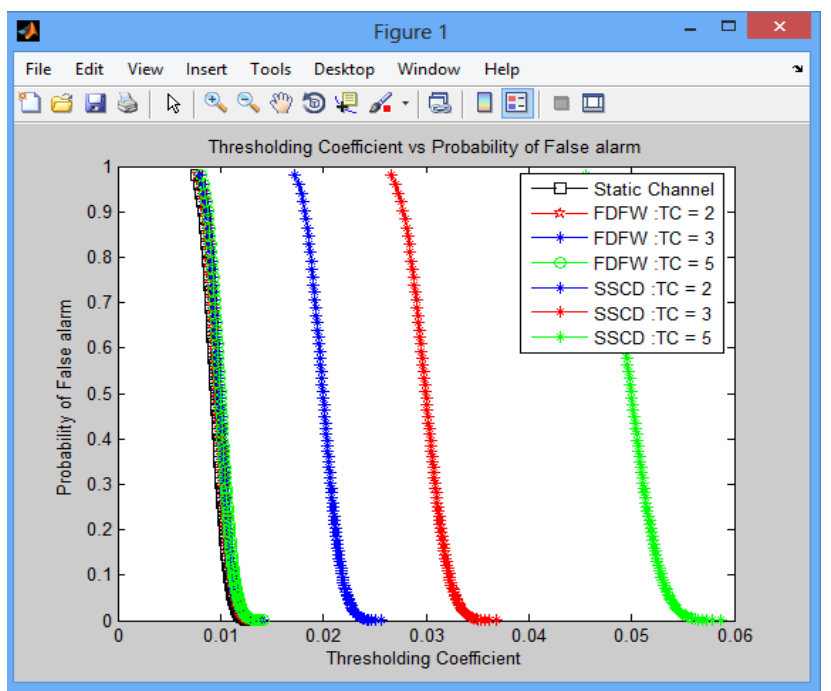

Fig. 5 Probability of false-alarm

If $\mathrm{P}_{\mathrm{f}}=0.1$ for example, we can obtain the corresponding detection thresholds for different sepration startegies of SSCD algorithm.

Fig. 6 and Fig. 7 shows the detection probablity of FDFW and SSCD algorithm, respectively.

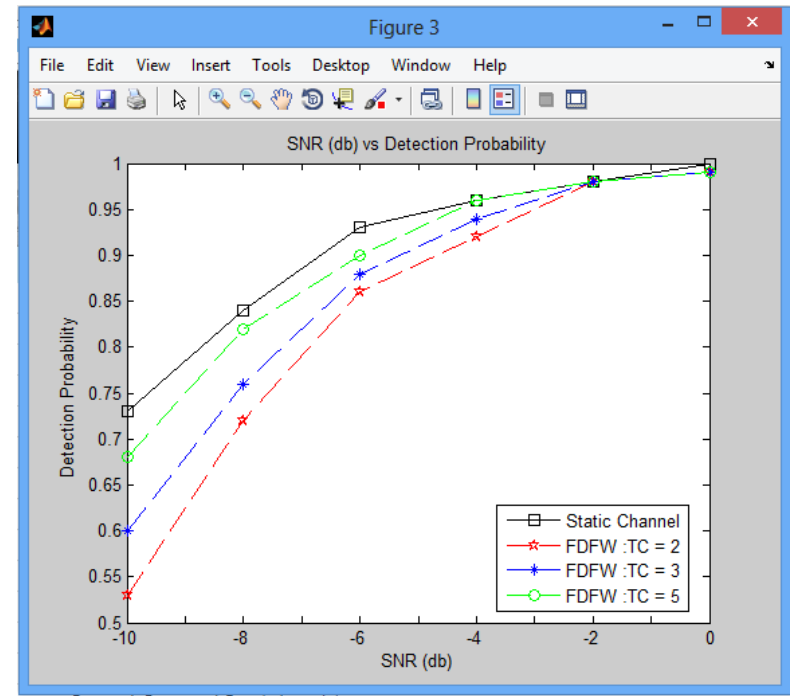

Fig. 6 Detection Probability in FDFW
In case of Fig 6, black curve is a benchmark showing detection probabitli under static channel case. Performance of FDFW deteriorates with the increase of channel varying rate.

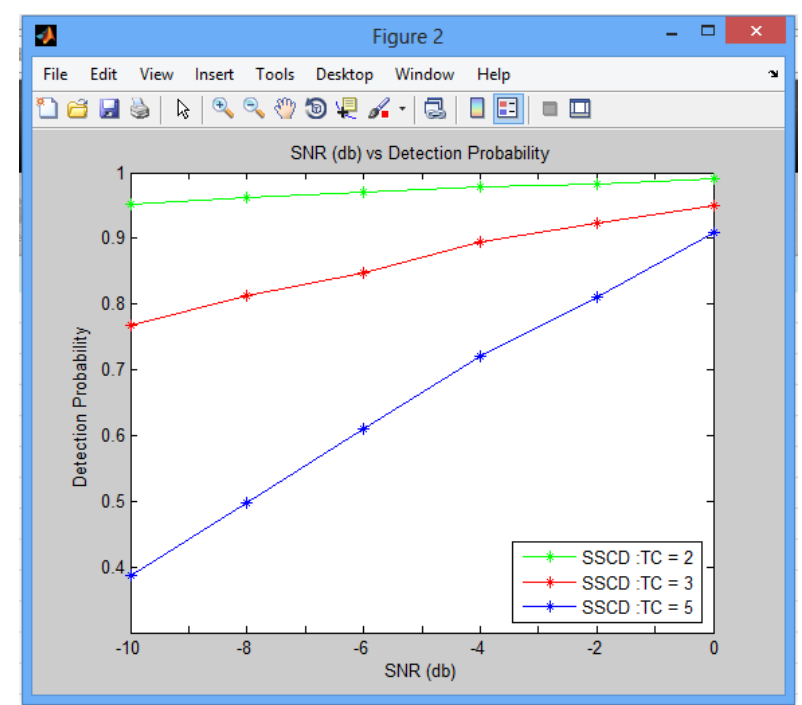

Fig. 7 Detection Probability in SSCD

In case of SSCD sensing accuracy id preserved under all given scenarios. Moreover, the SSCD algorithm induces the channel state diversity gain and the detection probability is improved. SSCD improves the negative effect of fast time varying channels.

\section{CONCLUSION}

In this we have proposed an algorithm SSCD which is very much effective in indentifying the features of primary users. By including channel-state diversity SSCD accumulates and captures feature branches with different phases during an observation window more accurately. Negative effect of fasttime varying channels is also shown on sensing process. Numerical results will prove that SSCD algorithm significantly outperforms traditional FDFW algorithm under fast time-varying channels.

Future work will include detailed derivation on the theoretical relationship of probability of false-alarm, window separation strategy and the detection threshold.

\section{REFERENCES}

[1] Tianheng $\mathrm{Xu}$,Mengying Zhang, Honglin Hu, Yang Yang, "Spectrum Sensing with channel state diversity", IEEE,2016.

[2] M. Sherman, A. N. Mody, R. Martinez, C. Rodriguez and R. Reddy, "IEEE standards supporting cognitive radio and networks, dynamic spectrum access, and coexistence," IEEE Commun. Mag., vol. 46, no. 7, pp. 72-79, Jul. 2008.

[3] S. Atapattu, C. Tellambura and H. Jiang, "Energy detection based cooperative spectrum sensing in cognitive radio networks,” IEEE Trans. Wireless Commun., vol. 10, no. 4, pp. 1232-1241, Apr. 2011.

[4] H. Qing, Y. Liu, G. Xie and J, Gao, "Wideband Spectrum Sensing for Cognitive Radios: A Multistage Wiener Filter Perspective,” IEEE Signal Process. Lett., vol. 22, no. 3, pp. 332335, Mar. 2015.

[5] B. Li, M. Sun M, X. Li, et al., "Energy Detection Based Spectrum Sensing for Cognitive Radios Over Time-Frequency 
Doubly Selective Fading Channels,” IEEE Trans. Signal Process., vol. 63, no. 2, pp. 402-417, Jan. 2015.

[6] Tianheng $\mathrm{Xu}$, Mengying Zhang, Honglin $\mathrm{Hu}$, and Yang Yang,"Spectrum sensing with channel state diversity", IEEE, 978-1-5090-0304-4, 2016.

[7] P. D. Sutton, K. E. Nolan, and L. E. Doyle, "Cyclostationary signatures in practical cognitive radio applications,” IEEE J. Sel. Areas Commun., vol. 26, no. 1, pp. 13-24, Jan. 2008.

[8] S. Shobana, R. Saravanan and R. Muthaiah, "Matched filter based spectrum sensing on cognitive radio for OFDM WLANs," Int. J. Eng.Technol., vol. 5, no. 1, pp. 142-146, Feb.-Mar. 2013.

[9] H. Tang, "Some physical layer issues of wide-band cognitive radio systems," in Proc. IEEE DySPAN 2005, pp. 151-159.

[10] A. Zahedi-Ghasabeh, A. Tarighat and B. Daneshrad, "Spectrum sensing of OFDM waveforms using embedded pilots in the presence of impairments,” IEEE Trans. Veh. Technol., vol. 61, no. 3, pp. 1208-1221, Mar.2012

[11] E. Axell, G. Leus, E. G. Larsson and H. V Poor, "Spectrum sensing for cognitive radio: State-of-the-art and recent advances,” IEEE Signal Process. Mag., vol. 29, no. 3, pp. 101116, May. 2012

[12] T. Yucek and H. Arslan, "A survey of spectrum sensing algorithms for cognitive radio applications,” IEEE Commun. Surveys Tuts., vol. 11, no. 1, pp. 116-130, First Quarter 2009.

[13] A. Osseiran, F. Boccardi, V. Braun, et al., "Scenarios for 5G mobile and wireless communications: the vision of the METIS project,” IEEE Commun. Mag., vol. 52, no. 5, pp. 26-35, May. 2014. 